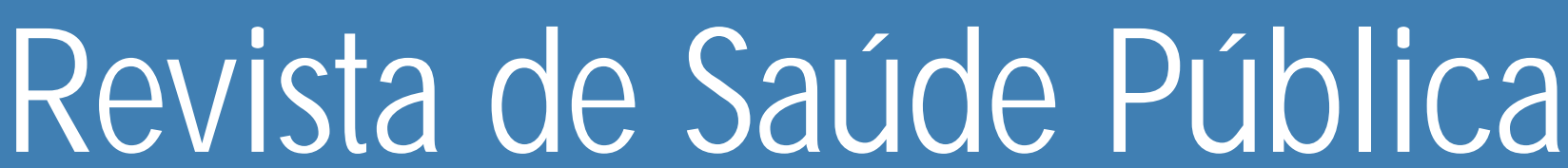

\begin{tabular}{lllllll}
\hline & 0 & $U$ & $R$ & $N$ & $A$ & $L$
\end{tabular}

$0 F$

P U B L I C

H E A L T H

\title{
Tabagismo em gestantes de área urbana da região Sul do Brasil, 1982 e 1993
}

\section{Tobacco smoking among pregnant women in an urban area in Southern Brazil, 1982-93}

Bemardo Lessa Horta, Cesar G omes Victora, Fernando C. Barros, Ina da Silva dos Santos e Ana M. B. Menezes

U niversidade Católica de Pelotas. Pelotas, RS - Brasil (B.L.H.); Departamento de Medicina Social da Universidade Federal de Pelotas. Pelotas, RS - Brasil (C.G.V., F.C.B., I.S.S.); Departamento de Clínica Médica da U niversidade Federal de Pelotas. Pelotas, RS - Brasil (A.M.B.M.) 


\title{
Tabagismo em gestantes de área urbana da região Sul do Brasil, 1982 e 1993
}

\section{Tobacco smoking among pregnant women in an urban area in Southern Brazil, 1982-93}

\author{
Bemardo Lessa Horta, Cesar G omes Victora, Fernando C. Barros, Ina da Silva dos Santos e \\ Ana M. B. Menezes \\ U niversidade Católica de Pelotas. Pelotas, RS - Brasil (B.L.H.); Departamento de Medicina Social da \\ Universidade Federal de Pelotas. Pelotas, RS - Brasil (C.G.V., F.C.B., I.S.S.); Departamento de Clínica \\ Médica da Universidade Federal de Pelotas. Pelotas, RS - Brasil (A.M.B.M.)
}

\begin{abstract}
Resumo
Objetivo Avaliar as mudanças na prevalência de tabagismo durante a gravidez na cidade de Pelotas, RS, Brasil, com base nos estudos da população materno-infantil realizados nos anos de 1982 e 1993.
Metodologia
Estudo transversal, tendo sido identificados 6.011 e 5.304 recém-nascidos, cujas famílias residiam na área urbana da cidade de Pelotas, respectivamente em 1982 e 1993.

Resultados O tabagismo materno durante a gestação apresentou uma discreta redução de $35,7 \%$, em 1982, para 33,5\% em 1993 (p < 0,05). O hábito de fumar esteve inversamente relacionado com a renda e o número de consultas no pré-natal.

Tabagismo, epidemiologia. Gravidez.

Abstract

Objective Acomparison between on the prevalence of smoking during pregnancy in 1982 and that in 1993 in Pelotas, Southern Brazil.

Methodology Cross-sectional study. All hospital deliveries in 1982 and 1993 - corresponding to over $99 \%$ of all births in those years - were studied. A total of 6,011 and 5,304 mothers were interviewed, respectively.

Results The prevalence of smoking during pregnancy showed a small decrease from $35.7 \%$ in 1982 to $33.5 \%$ in 1993 ( $p<0.05$ ). In the two years under study, family income and number of antenatal care visits were inversely associated with the prevalence of maternal smoking. The rate of stopping smoking during pregnancy was $20.6 \%$.
\end{abstract}

Smoking, epidemiology. Pregnancy.

Correspondência para/Correspondence to: Bernardo Lessa Horta - Rua Anchieta, 4043 - 96015-420 Pelotas - RS - Brasil.

E mail: blhorta@phoenix.ucpel.tche.br

Recebido em 22.8.1996. Aprovado em 31.1.1997. 


\section{INTRO DUÇÃO}

Os prejuízos causados à saúde pelo hábito de fumar são amplamente conhecidos ${ }^{6,12}$, sendo o seu controle considerado pela Organização Mundial da Saúde (OMS) como o maior desafio da saúde pública no mundo atual ${ }^{17}$. O controle do vício tabágico pode fazer mais pela saúde do homem e sua expectativa de vida do que qualquer outra ação preventiva isolada ${ }^{19}$.

Até o início da década passada observou-se, em vários países, uma redução importante do tabagismo entre as mulheres ${ }^{18,20}$. Entretanto, nos Estados Uni$\operatorname{dos}^{3}$ a prevalência estabilizou-se em $22,5 \%$ entre 1990 e 1993. Fenômeno semelhante foi descrito para uma região do Canadás. Infelizmente não se dispõe de dados representativos sobre a evolução do tabagismo no Brasil.

Durante a gravidez, o tabagismo materno aumenta o risco de baixo peso ao nascer ${ }^{15}$ e de retardo do crescimento intrauterino ${ }^{10}$, como também, a morbi-mortalidade fetal ${ }^{13}$. Em virtude de estes e de outros riscos, um dos objetivos do governo dos Estados Unidos, na área da saúde, é a redução da prevalência do tabagismo entre as mulheres em idade fértil para $12 \%$, no ano $2000^{2}$.

O presente estudo teve como objetivo avaliar as mudanças na prevalência de tabagismo durante a gravidez, na cidade de Pelotas, RS, com base nos estudos da população materno-infantil realizados nos anos de 1982 e 1993.

\section{METO DO LOG IA}

Como parte do Estudo Longitudinal da População Materno-Infantil de Pelotas, foram avaliados todos os nascimentos hospitalares ocorridos nos anos de 1982 e 1993. As cinco maternidades da cidade foram visitadas diariamente e as parturientes entrevistadas logo após o parto. Em 1982, dos 7.398 nascimentos hospitalares foi possível realizar entrevista em 7.392. Em 1993, foram entrevistadas 6.394 mães, tendo havido sete perdas e nove recusas. Entre as mães entrevistadas, foram selecionadas para o estudo aquelas que residiam na área urbana de Pelotas. A metodologia destes estudos encontra-se descrita em outras publicações ${ }^{1,21,22}$.

Em dois dos citados estudos ${ }^{1,22}$ os entrevistadores eram estudantes de medicina, previamente treinados. Cerca de $5 \%$ das entrevistas foram repetidas pelos supervisores para garantir a qualidade do trabalho de campo. Utilizou-se um questionário padronizado com perguntas sobre características socioeconômicas e demográficas, utilização de serviços de pré-natal e sobre o tabagismo materno na gravidez, entre outras variáveis.
Considerou-se como fumante toda mulher que informou ter fumado durante a gravidez, independentemente do número de cigarros. A taxa de abandono do tabagismo foi obtida através da divisão do número de mulheres que interromperam o hábito pelo número de fumantes no início da gravidez.

Foi utilizado o teste do qui-quadrado para a comparação entre proporções ${ }^{14}$. $\mathrm{O}$ ajuste para possíveis fatores de confusão foi feito através da regressão logística, tendo sido mantidas na análise apenas as variáveis cuja inclusão alterou em pelo menos $10 \%$ a medida de efeito (razão de odds) ${ }^{16}$.

\section{RESU LTAD OS}

Foram estudados 6.011 e 5.304 mães residentes na área urbana da cidade de Pelotas, respectivamente em 1982 e 1993.

A Tabela 1 mostra a distribuição da população estudada de acordo com algumas características maternas. Observa-se entre 1982 e 1993, uma redução nas proporções de mães com renda familiar menor ou igual a um salário-mínimo, e de mães sem escolaridade. No que diz respeito à idade materna, houve um discreto aumento nos grupos extremos (abaixo de 20 anos e acima de 34 anos). Não se observou aumento na cobertura do programa de prénatal, mas entre aquelas mães que fizeram pré-natal houve importante aumento no número de consultas. Em 1982, 76,7\% das mães tiveram cinco ou mais consultas de pré-natal, enquanto que em 1993 esta proporção aumentou para $82,2 \%$. O percentual de gestantes com dez ou mais consultas duplicou.

O tabagismo materno durante a gestação apresentou uma pequena redução, de 35,7\% em 1982 para 33,5\% em 1993 ( p < 0,05). No tocante à intensidade do tabagismo, não se observou variação importante na década: em 1982, 8,6\% das gestantes fumaram 15 ou mais cigarros por dia durante a gravidez; em 1993, esse percentual foi de 7,3\%. Em 1982, 8,1\% das gestantes pararam de fumar durante a gravidez; para 1993, esse percentual foi de $6,9 \%$. Considerando apenas as gestantes tabagistas, a taxa de abandono do tabagismo durante a gravidez foi de $22,7 \% \mathrm{e}$ $20,6 \%$, respectivamente (Tabela 2 ).

Em 1982, a idade materna esteve inversamente relacionada com a prevalência de tabagismo: $41 \%$ das mães com idade menor de 20 anos eram fumantes, enquanto que naquelas com 40 anos ou mais esta prevalência foi de 25,2\% (p para tendência linear < 0,001). Entretanto, em 1993, não se observou tal associação: o tabagismo foi menos freqüente nos grupos extremos de idade (abaixo de 20 anos e acima de 40 anos) (Figura 1). Quando as prevalências de fumo foram 
Tabela 1 - Distribuição da população estudada de acordo com algumas características maternas. Pelotas, 1982 e 1993.

Table 1 - Distribution of study population according to maternal variables. Pelotas, 1982 and 1993.

\begin{tabular}{|c|c|c|}
\hline \multirow{2}{*}{ Características maternas } & \multicolumn{2}{|c|}{ População estudada } \\
\hline & $1982 *(\%)$ & 1993** (\%) \\
\hline \multicolumn{3}{|c|}{ Renda familiar em salários-mínimos \# } \\
\hline$\leq 1$ & 22,1 & 19,0 \\
\hline $1,1-3$ & 47,4 & 41,7 \\
\hline $3,1-6$ & 18,5 & 23,5 \\
\hline $6,1-10$ & 6,4 & 8,4 \\
\hline$>10$ & 5,6 & 7,4 \\
\hline \multicolumn{3}{|l|}{ Escolaridade materna \# } \\
\hline Sem escolaridade & 5,6 & 2,6 \\
\hline $1-4$ anos & 27,8 & 25,7 \\
\hline $5-8$ anos & 41,6 & 46,2 \\
\hline$\geq 9$ anos & 25,0 & 25,5 \\
\hline \multicolumn{3}{|l|}{ Idade materna (anos) \# } \\
\hline$<20$ & 15,3 & 17,4 \\
\hline $20-24$ & 31,1 & 27,6 \\
\hline $25-29$ & 27,0 & 25,7 \\
\hline $30-34$ & 16,6 & 18,2 \\
\hline $35-39$ & 7,6 & 8,9 \\
\hline$\geq 40$ & 2,4 & 2,2 \\
\hline \multicolumn{3}{|c|}{ Número de consultas no pré-natal \# } \\
\hline $\mathrm{N}$ ão consultou & 5,1 & 4,9 \\
\hline 1 - 4 vezes & 18,3 & 13,0 \\
\hline $5-9$ vezes & 62,1 & 53,9 \\
\hline$\geq 10$ vezes & 14,6 & 28,3 \\
\hline
\end{tabular}

examinadas conforme o ano de nascimento das mães, observa-se uma queda importante para as mães nascidas entre 1958 e 1967, que em 1993 fumavam menos do que em 1982. Para as mães nascidas entre 1953 e 1957, não houve variação (Figura 2).

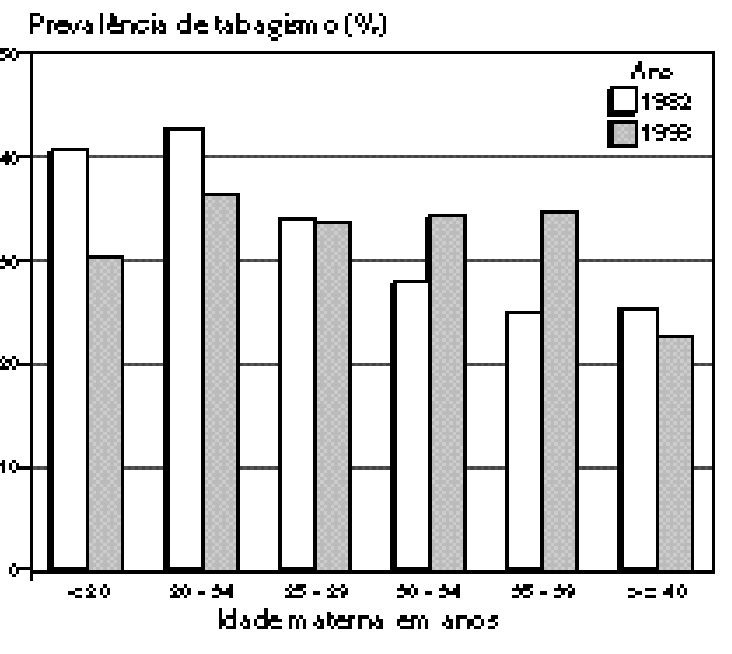

Figura 1 - Prevalência de tabagismo materno na gravidez de acordo com a idade. Pelotas, 1982 e 1993.

Figure 1 - Prevalence of tobacco smoking during pregnancy by age. Pelotas, 1982 and 1993.
A Tabela 3 mostra que, em ambos os anos, a prevalência de tabagismo foi menor nas mães de alta renda ou com escolaridade de nove anos ou mais. Por outro lado, observou-se um aumento na prevalência de tabagismo no grupo com menos de

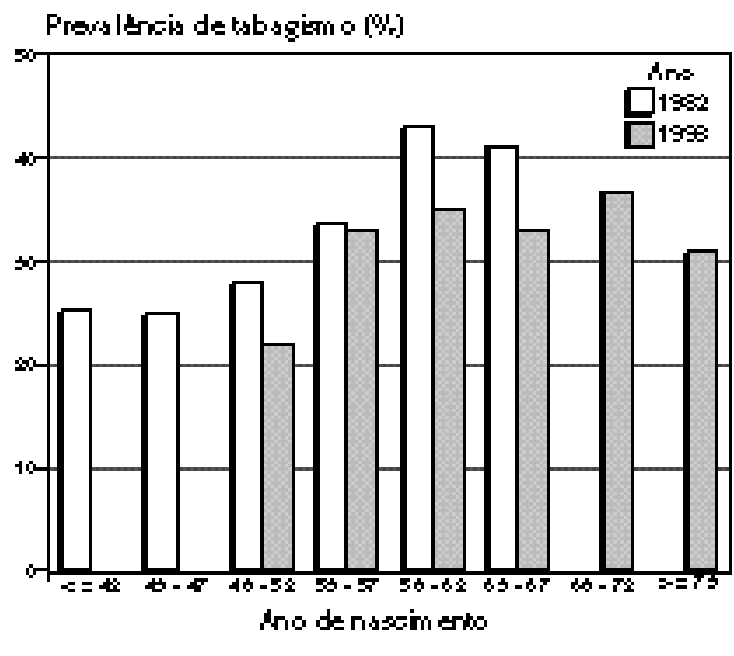

Figura 2 - Prevalência de tabagismo materno na gravidez de acordo com o ano de nascimento. Pelotas, 1982 e 1993.

Figure 2 - Prevalence of tobacco smoking during pregnancy by year of birth. Pelotas, 1982 and 1993. 
Tabela 2 - Prevalência de tabagismo de acordo com o ano. Pelotas, 1982 e 1993.

Table 2 - Prevalence of maternal smoking during pregnancy by year of birth. Pelotas, 1982 and 1993.

\begin{tabular}{|c|c|c|}
\hline \multirow{2}{*}{ Tabagismo } & \multicolumn{2}{|c|}{ Prevalência } \\
\hline & $1982 *(\%)$ & $1993 * *(\%)$ \\
\hline Tabagismo materno na gestação \# & 35,7 & 33,5 \\
\hline $\begin{array}{l}\text { Duração do tabagismo na gravidez \# } \\
\text { Não fumou } \\
\text { Parou durante a gravidez } \\
\text { Fumou toda gravidez }\end{array}$ & $\begin{array}{r}64,3 \\
8,1 \\
27,6\end{array}$ & $\begin{array}{r}66,5 \\
6,9 \\
26,6\end{array}$ \\
\hline $\begin{array}{l}\text { Número de cigarros \# } \\
\quad \text { Não fumou } \\
\quad<15 \text { cig/dia parcialmente } \\
<15 \text { cig/dia toda gravidez } \\
\geq 15 \mathrm{cig} / \text { dia }\end{array}$ & $\begin{array}{r}64,3 \\
7,4 \\
19,7 \\
8,6\end{array}$ & $\begin{array}{r}66,5 \\
6,3 \\
19,9 \\
7,3\end{array}$ \\
\hline
\end{tabular}

$* \mathrm{~N}=6.011$

$* * \mathrm{~N}=5.304$

$\# p<0,05$ na comparação entre 1982 e 1993.

quatro anos de escolaridade. A freqüência ao prénatal esteve marcadamente associada com a prevalência de tabagismo, sendo este quase duas vezes mais freqüente naquelas mães que não fizeram prénatal em relação às que consultaram dez ou mais vezes. Esta relação se manteve mesmo após ajuste para as variáveis renda familiar, escolaridade e idade materna, como mostram as razões de "odds" ajustadas na Figura 3.

\section{Raz5o de "odde"pars lumo ns getsç5o}

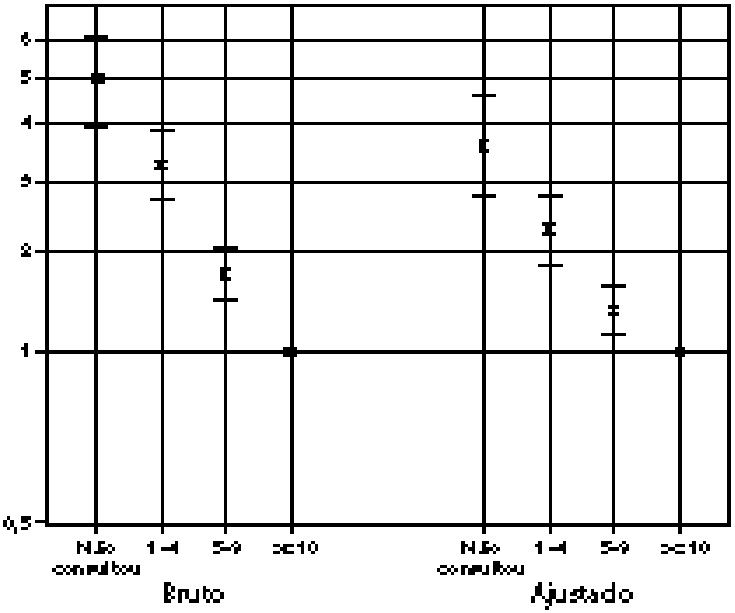

Nimero de conaskss no prë-nstsl

Nustsdopars: reads bm iliar, exolaridsde $e$ idsde materns.

Figura 3 - Tabagismo durante a gravidez de acordo com a freqüência ao pré-natal. Pelotas, 1982 e 1993.

Figure 3 - Prevalence of tobacco smoking during pregnancy by number of antenatal care visits. Pelotas, 1982 and 1993.
Observou-se ainda uma relação inversa entre o número de consultas pré-natais e a taxa de abandono do tabagismo. Entre as fumantes que não fizeram prénatal, apenas 13,6\% pararam de fumar, enquanto que entre aquelas que consultaram dez ou mais vezes esse percentual foi de $25,0 \%$. Este efeito se manteve mesmo após ajuste para os seguintes fatores de confusão: renda familiar, idade materna, cor da pele e paridade (Figura 4).

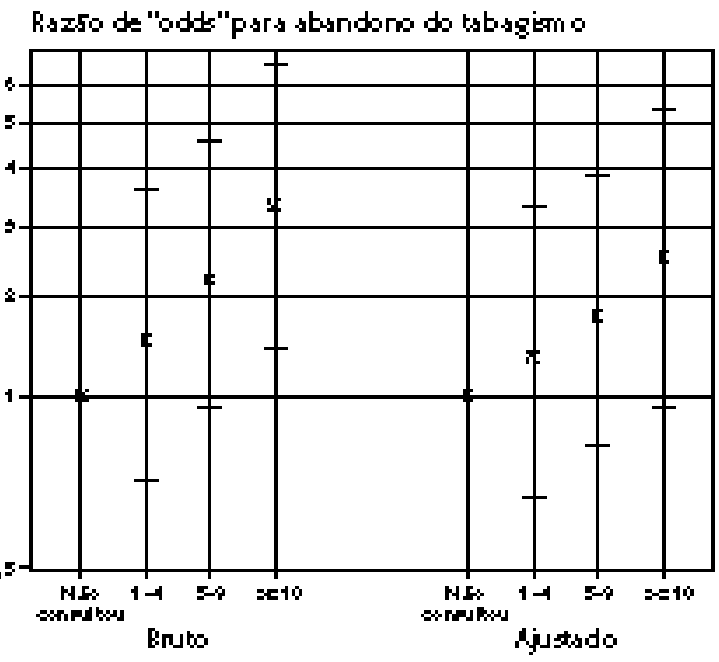

Nimero de conaukss no prë-nstsl

Mustadopars: reads am ilisr, idsde materns, or epsridsde.

Figura 4 - Risco de abandono do tabagismo durante a gravidez de acordo com a freqüência ao pré-natal. Pelotas, 1982 e 1993.

Figure 4 - 0 dds ratio for stopping smoking during pregnancy by number of antenatal care visits. Pelotas, 1982 and 1993. 
Tabela 3 - Tabagismo materno na gestação de acordo com variáveis socioeconômicas, demográficas e de freqüência ao prénatal. Pelotas, 1982 e 1993.

Table 3 - Maternal smoking during pregnancy by socioeconomic and demographic variables and number of antenatal care visits. Pelotas, 1982 and 1993.

\begin{tabular}{lcc}
\hline \multirow{2}{*}{ Variáveis socioeconômicas } & \multicolumn{2}{c}{ Prevalência de tabagismo } \\
\cline { 2 - 3 } & $1982(\%)$ & $1993(\%)$ \\
\hline Renda familiar em salários-mínimos & $\#$ & $\#$ \\
$\leq 1$ & 44,0 & 30,3 \\
$1,1-3$ & 36,5 & 28,6 \\
$3,1-6$ & 32,5 & 24,7 \\
$6,1-10$ & 20,4 & 22,8 \\
$>10$ & 25,0 & $\#$ \\
Escolaridade materna & $\#$ & 45,3 \\
Sem escolaridade & 37,6 & 42,2 \\
$1-4$ anos & 37,8 & 34,2 \\
$5-8$ anos & 38,5 & 22,2 \\
$\geq 9$ anos & 28,3 & $\#$ \\
Freqüência ao pré-natal & $\#$ & 48,2 \\
Não consultou & 55,7 & 42,6 \\
$1-4$ consultas & 44,1 & 33,8 \\
5 - consultas & 33,4 & 26,0 \\
\hline 10 consultas & 28,4 & 33,5 \\
\hline Total & 35,7 & \\
\hline
\end{tabular}

$\# p<0,05$ na comparação dentro de cada coluna.

Tabela 4 - Taxa de abandono ao tabagismo durante a gestação de acordo variáveis socioeconômicas, demográficas e de freqüência ao pré-natal. Pelotas, 1982 e 1993.

Table 4 - Rate of stopping smoking during pregnancy by socioeconomic and demographic variables and number of antenatal care visits. Pelotas, 1982 and 1993.

\begin{tabular}{lcc}
\hline \multirow{2}{*}{ Variáveis socioeconômicas } & \multicolumn{2}{c}{ Prevalência de tabagismo } \\
\cline { 2 - 3 } & $1982(\%)$ & $1993(\%)$ \\
\hline Renda familiar em salários-mínimos & & $\#$ \\
$\leq 1$ & 20,0 & 16,1 \\
$1,1-3$ & 22,2 & 18,6 \\
$3,1-6$ & 25,5 & 25,9 \\
$6,1-10$ & 31,9 & 27,9 \\
$>10$ & 27,2 & 29,4 \\
Escolaridade materna & $\#$ & 21,0 \\
Sem escolaridade & 16,7 & 19,4 \\
$1-4$ anos & 19,3 & 19,9 \\
$5-8$ anos & 22,8 & 24,8 \\
$\geq 9$ anos & 29,7 & $\#$ \\
Idade materna & & 26,7 \\
$<20$ & 24,9 & 20,2 \\
$20-24$ & 23,2 & 20,4 \\
$25-29$ & 22,2 & 15,4 \\
$30-34$ & 23,1 & 20,9 \\
$35-39$ & 18,6 & 26,0 \\
\hline 40 & 10,7 & 20,6
\end{tabular}

\# $p<0,05$ na comparação dentro de cada coluna.

Em ambos os períodos, o abandono do tabagismo durante a gravidez foi mais comum entre as mulheres com melhor nível socioeconômico. Em 1982, as mulheres mais jovens pararam de fumar em maior proporção do que as demais; em 1993, não se observou tal tendência, sendo o abandono do hábito de fumar mais comum nos grupos extremos de idade (Tabela 4).

\section{DISCU SSÃO}

Os estudos de base populacional realizados em 1982 e 1993 cobriram mais de $99 \%$ dos nascimentos hospitalares ocorridos na cidade de Pelotas. Os nascimentos domiciliares são muito $\operatorname{raros}^{1}$, sendo mínima, portanto, a possibilidade de viés de seleção. Os 
estudos permitiram a comparação da evolução do tabagismo materno, durante a gravidez, em um período de 11 anos, contribuindo para a avaliação do possível impacto do Programa Nacional de Combate ao Fumo, iniciado em $1985^{11}$.

Uma associção inversa entre idade e hábito do tabagismo havia sido observada tanto em um estudo de base populacional, realizado em Pelotas em $1988,{ }^{9}$ quanto no presente estudo referente ao ano de 1982, indicando maior percentual de fumantes entre mulheres jovens; já em 1993, não se observou tal relação. A análise conforme o ano de nascimento das mães mostra que as maiores prevalências ocorreram nas mães que nasceram entre 1958 e 1967. Esta tendência, se mantida, poderá levar a uma maior redução na prevalência do tabagismo nos próximos anos.

O aumento na prevalência de tabagismo no grupo com menor escolaridade também tem sido observado em outros países, o que, provavelmente, se deva a um menor acesso dessas mulheres às informações sobre os riscos do fumo ${ }^{4}$.

A taxa de abandono do tabagismo durante a gestação, em ambos os anos, foi inferior à observada nos Estados Unidos 7 , em 1985 (39,3\%). No presente estudo, no ano de 1993, as mulheres nos grupos extremos de idade $(<20$ anos, $>=40$ anos $)$ apresentaram as maiores taxas de abandono de tabagismo, sendo que cerca de uma em cada quatro dessas abandonaram o hábito durante a gravidez. Resultado semelhante foi observado em outro estudo realizado na cidade de Pelotas, em $1989^{8}$.

\section{REFERÊN CIAS BIBLIO GRÁFICAS}

1. BARROS, F.C.; VICTORA, C.G.; VAUGHAN, J.P. The Pelotas birth cohort study, 1982-1987: strategies for following up, 6,000 children in a developing country. Paediatr. Perinatal Epidemiol., 4:267-82, 1990.

2. CENTER FOR DISEASE CONTROL. Cigarette smoking among women of reproductive age - United States, 19871992. MMWR, 43:789-91,1994.

3. CENTER FOR DISEASE CONTROL. Cigarette smoking among adults - United States, 1993. JAMA, 369:273, 1995.

4. CHOLLAT-TRAQUET, C. La mujer y el tabaco. Ginebra, Organización Mundial de la Salud, 1993.

5 DODDS, L. Prevalence of smoking among pregnant women in Nova Scotia from 1988 to 1992. Can. Med .Assoc. J., 152:185-90, 1995.

6 DOLL, R. Tobacco: an overview of health effects. In: Zaridze, D. \& Peto, R. Tobacco: a major health hazard. Lyon, International Agency for Research on Cancer, 1986. p. 11-22.
Fica a questão se a relação inversa entre a taxa de abandono do tabagismo e o número de consultas no pré-natal seria conseqüência do próprio pré-natal, ou de um viés de auto-seleção. As mulheres com maior número de consultas já iniciaram o pré-natal fumando menos, havendo esta relação se mantido mesmo após ajuste para um possível efeito de confusão das variáveis socioeconômicas e demográficas. Portanto, algumas características ou atitudes maternas podem não somente determinar o hábito de fumar, como também a procura pelo serviço de pré-natal. A possibilidade de auto-seleção, portanto, não pode ser descartada.

Tendo em vista a quase universalidade do atendimento pré-natal em Pelotas e em outras áreas urbanas do Brasil, a gravidez deve ser vista como o momento ideal para o abandono do tabagismo. Nesse período ocorre uma intensificação dos contatos com os profissionais de saúde, propiciando, assim, uma oportunidade para que se estimule o abandono desse hábito, salientando seus possíveis malefícios sobre a saúde da criança. É preocupante o fato de que as mulheres de menor nível educacional, que justamente são aquelas que menos utilizam os serviços de pré-natal ${ }^{21}$, sejam aquelas que mais fumam, cuja prevalência de tabagismo aumentou na década e que menos abandonaram o hábito na gestação. Uma vez que seus filhos, independemente do tabagismo, já apresentam maior risco de baixo peso ao nascer, retardo do crescimento intrauterino e mortalidade infantil ${ }^{21}$, é fundamental que os serviços de saúde dediquem atenção prioritária a estas gestantes.

7. FINGERHUT, L.A.; KLEINMAN, J.C.; KENDRICK, J.S. Smoking before, during and after pregnancy. AJPH, 80:5414, 1990.

8. HALAL, I.S.; VICTORA, C.G.; BARROS, F.C. Determinantes do hábito de fumar e de seu abandono durante a gestação em localidade urbana da região Sul do Brasil. Rev. Saúde Pública, 27:105-12, 1993.

9. HORTA, B.L.; RAMOS, E.O.; VICTORA, C.G. Determinantes do hábito de fumar na cidade de Pelotas, Brasil. Bol. Ofic. Sanit Panamer., 113:131-6, 1992.

10. HORTA, B.L.; VICTORA, C.G.; MENEZES, A.M.; HALPERN, R.; BARROS, F.C. Low birthweight, preterm births and intrauterine growth retardation in relation to maternal smoking. Paediatr. Perinatal Epidemiol., 11:140-51, 1997.

11. INSTITUTO NACIONAL DE CÂNCER. Controle do tabagismo um desafio. Rio de Janeiro, Instituto Nacional de Câncer, 1992. 
12 INTERNATIONAL AGENCY FOR RESEARCH ON CANCER. (IARC) Monographs on evaluation of the carcinogenic risk of chemicals to humans: tobacco smoking. Lyon, 1986. (IARC Scientific Publication 38).

13. KINE, J.; STEIN, Z.A.; SUSSER, M.; WARBURTON, D. Smoking: a factor for spontaneous abortion. N. Engl. J. Med., 297:793-6, 1977.

14. KIRKWOOD, B.R. Essentials of medical statistics. Oxford, Blackwell Scientific Publications, 1988.

15. KRAMER, M.S. Determinants of low birth weight: methodological assessment and meta-analysis. Bull. World Health Organ., 65:663-737, 1987.

16. MALDONADO, G. \& GREENLAND, S. Simulation study of confounders-selection strategies. Am. J. Epidemiol., 138:923-36, 1993.

17. ORGANIZACIÓN MUNDIAL DE LA SALUD.

Consecuencias del tabaco para la salud. Genebra, Organización Mundial de la Salud, 1974. (Série Informes Técnicos 568).
18. PIERCE, J.P. Progress and problems in international public health efforts to reduce tobacco usage. Annu. Rev. Public. Health, 12:383-400, 1991.

19. RAVENHOLT, R.T. Tobacco's impact on twentieth-century U.S. mortality patterns. Am. J. Prev. Med., 1:4-17, 1985.

20. TABAQUISMO EN LAS AMERICAS. Bol. Epidemiol. OPS, 10:1-5, 1989.

21. VICTORA, C.G.; BARROS, F.C.; VAUGHAN, J.P. Epidemiologia da desigualdade: um estudo longitudinal de 6.000 crianças brasileiras. $2^{\mathrm{a}}$ ed. São Paulo, CEBES/ HUCITEC, 1989.

22. VICTORA, C.G.; BARROS, F.C.; HALPERN, R.; MENEZES, A.M.B.; HORTA, B.L.; TOMASI, E.; WEIDERPASS, E.; CESAR, J.A.; OLINTO, M.T.; GUIMARÃES, P.R.V.; GARCIA, M.M.; VAUGHAN, J.P. Estudo longitudinal da população materno-infnatil da região urbana do Sul do Brasil, 1993: aspectos metodológicos e resultados preliminares. Rev. Saúde Pública, 30:34-45, 1996. 